\title{
Fijación vertebral posterior guiada por neuronavegación. Experiencia en 121 casos
}

\author{
R. Florensa*; J. Muñoz*; I. Cardiel; A. Bescós; M. Tardáguila; G. Plans*; C.J. Domínguez y S. Colet
}

Servicio de Neurocirugía Hospital Germans Trias i Pujol. Centro Médico Teknon*. Barcelona.

\section{Resumen}

Introducción. Los autores aportan una serie de 121 casos de instrumentación vertebral posterior realizadas desde septiembre del 2008 a diciembre del 2010 mediante la utilización de Fluoro 2D y TC asistida con neuronavegador Vector Visión o Kolibri. (Brain LAB, Feldkirchen, Germany).

Material. La muestra fue de 68 varones y 53 mujeres con edades comprendidas entre 24 y 75 años con una media de 50,35 años, todos ellos con indicación para una instrumentación vertebral posterior por distintas patologías.

Método. A todos se les realizó una TC previo al procedimiento según protocolo específico de adquisición de imagen para navegación, estas se fusionaron en el navegador con las adquiridas en el quirófano con un Fluoro 2D, lo cual permitió realizar una reconstrucción de alta calidad y así poder navegar de forma real-virtual. Para valorar los resultados se realizó una TC postoperatorio y se clasificó la posición del implante según la escala tomográfica de Heary. Se valoró también el tiempo de calibración del material y número de disparos con el Fluoro-2D. Los resultados clínicos se valoraron con las escalas de VAS, Oswestry y JOA (L) así como el grado de satisfacción con el procedimiento y aceptación del mismo.

Resultados. Se implantaron un total de 580 tornillos distribuidos en 62 cervicales ( 24 en C1-C2), 38 dorsales, 370 lumbares y 110 sacros. Se realizó cirugía abierta en 42 casos, MIS 28 y percutánea en 51. La precisión del implante fue del $\mathbf{9 8 , 4 5 \%}$ con una desviación global del $1,55 \%$ que según escala de Heary se distribuyeron en Grado II: 2 ( 1 cervical, 1 lumbar), grado III: 4 (1 cervical, 2 dorsales, 1 lumbar), grado IV: 3 (1 cervical, 2 lumbares). Tiempo medio global de calibración por procedimiento fue de 2'49" y la media de exposición fluoroscópica fue de 1 disparo a nivel cervical y dorsal y 2 lumbar. La valoración clínica al mes de 121 pacientes

Recibido: 16-10-10. Aceptado: 26-12-10. fue de 8.6/3.0 en el VAS, $68.0 \%$ / 23.0\% en Oswestry y 6,4/13,1 en JOA (L), dichos parámetros se mantuvieron estables a los 3 meses en 100 pacientes y a los 6 meses en 87 pacientes respectivamente, el grado de satisfacción entre completamente y muy satisfechos fue en el $94.9 \%$ y aceptarían de nuevo el tratamiento más de un $94 \%$.

Conclusión. La navegación con fluoro-2D-TC es una técnica de muy alta precisión que disminuye complicaciones, el número de reintervenciones, la exposición de irradiación y el tiempo quirúrgico.

PALABRAS CLAVE. Navegación vertebral. Cirugía guiada. Fijación vertebral percutánea. Tornillos pediculares

Posterior spinal instrumentation image guided and assisted by neuronavigation. Experience in 120 cases

\section{Summary}

Introduction. The authors present the results of a series of 121 cases of posterior vertebral fixation carried out from Sept 2008 to Sept 2010 using Flouro 2D-TC assisted Vector Vision o Kolibri navigator. ( Brain LAB, Feldkirchen, Germany).

Material. The sample included 68 males and 53 females. Age range was 24-75 with an average of 50.35., all with indication for instrumentation by different pathologies.

Method. Patients presenting vertebral lesions of varying ethiology and lesion level with vertebral posterior fixation indication were included in the study. All underwent a CT before surgery, according to navigation protocol, and the images obtained were merged in the navigator with those obtained in the operating room with a Flouro $2 D$, which allowed a high quality $3 D$ reconstruction to be performed and thus the capacity to navigate in a real-virtual manner. To evaluate the results of the implant a post-op CT was performed and the position of the implant was defined according to the Heary scale. The calabration time of the material was also evaluated, number of shots with the Flouro-2D, 
and for clinical evaluation VAS scales were employed, Oswestry and JOA (L), as well as the degree of satisfaction and acceptance of the procedure.

Results. A total of 580 screws were implanted, distributed in 62 cervicals of which 24 were in C1-C2, 38 dorsals, 370 lumbar and 110 sacral. Open surgery was performed in 42 cases, MIS in 28 and percutaneous in 51. The presision of the implant was $98.45 \%$ with a global deviation of $1.55 \%$, that according to the Heary scale was distributed in grade ll: 2 (1 cervical, 1 lumbar) grade III: 4 ( 1 cervical, 2 dorsal, 1 lumbar), grade IV: 3 (1 cervical, 2 lumbar). General average time of calibration per procedure was $2 \mathrm{~min} .49$ seconds and the mean flouroscopic exposure was one shot at cervical and dorsal and two shots at lumbar level. The clinical evaluation at one month of 121 patients was 8.6/3.0 in the VAS, $68.0 \%$ $/ 23.0 \%$ in Oswestry and 6.4/13.1 in JOA $(\mathrm{L})$, with those parameters remaining stable at 3 months in 100 and at 6 months in 87 patients respectively, and the degree of satisfaction between being completely and very satisfied with the procedure was $94.9 \%$, and those who would submit to another treatment was more than $94 \%$.

Conclusion. Navigation with Flouro-2D-CT is a high precision technique that reduces complications of varying severity according to the level operated well as number of reinterventions, radiation exposure and surgical time.

KEY WORDS. Vertebral navigation. Guided surgery. Percutaneous vertebral fixation. Pedicle screws.

\section{Introducción}

La instrumentación vertebral posterior es una técnica con alta eficacia o refrendada en la literatura para el tratamiento de patologías degenerativas, traumáticas y

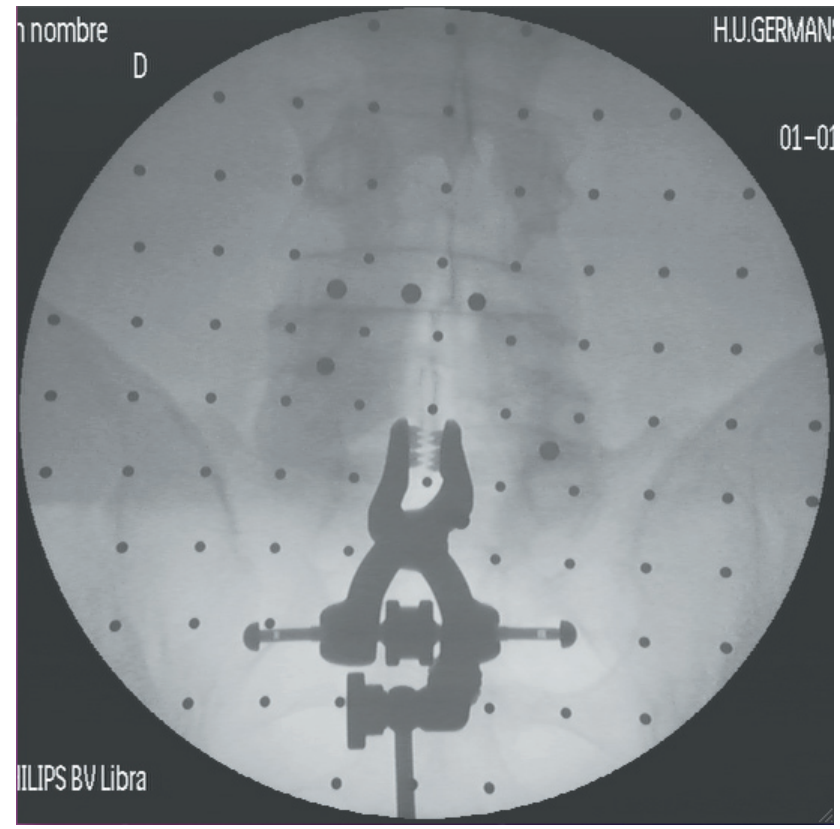

Figura 1. Rx. Ap. Con marcas referenciales y pinza en espinosa a intervenir.

tumorales. Se trata de una técnica avalada por un alto nivel de evidencia científica, pero no exenta de complicaciones, siendo estas mínimas en manos expertas pero que pueden conllevar gravedad importante según el nivel de la inter-

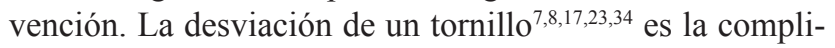
cación más reportada con porcentajes del 12 hasta un $40 \%$, de los cuales solo entre un 4 y $12 \%$ dan sintomatología clínica.

Clásicamente la instrumentación posterior se ha realizado según unas referencias anatómicas vertebrales 6 con el soporte del fluoroscopio 2D intra-operatorio. Con el objetivo de mejorar la precisión del implante, se han aportado diferentes métodos como cirugía guiada con Fra-

Tabla 1

Esquema comparativo entre los diferentes sistemas de navegación espinal

\begin{tabular}{|l|l|l|l|}
\hline Método & Fluoro TC y navegación & $\begin{array}{l}\text { Fluoro isocentric (3D) } \\
\text { con navegación }\end{array}$ & $\begin{array}{l}\text { Fluoro 2D virtual con } \\
\text { navegación fluoroscópica }\end{array}$ \\
\hline Imagen & $\begin{array}{l}3 \mathrm{D}, \text { alta calidad de la } \\
\text { anatomía ósea }\end{array}$ & $\begin{array}{l}3 \mathrm{D}, \text { limitada u un volumen de } \\
120 \mathrm{~mm}^{3}, \text { limitada en obesos }\end{array}$ & $2 \mathrm{D}$, limitada a obesos \\
\hline Preoperatorio & $\begin{array}{l}\text { TC obligatorio. Alta } \\
\text { resolución de la } \\
\text { reconstrucción }\end{array}$ & $\begin{array}{l}\text { No requiere imágenes } \\
\text { preoperatorias }\end{array}$ & $\begin{array}{l}\text { No requiere imágenes } \\
\text { preoperatorias }\end{array}$ \\
\hline Intraoperatorio & $\begin{array}{l}\text { Registro manual. No } \\
\text { corrige errores }\end{array}$ & $\begin{array}{l}\text { Registro automático, } \\
\text { corrección de errores }\end{array}$ & $\begin{array}{l}\text { Múltiples imágenes, } \\
\text { registro automatico, } \\
\text { tiempo quirúrgico largo }\end{array}$ \\
\hline Postoperatorio & Puede ser necesario un TC & No necesario TC & Puede ser necesario TC \\
\hline
\end{tabular}




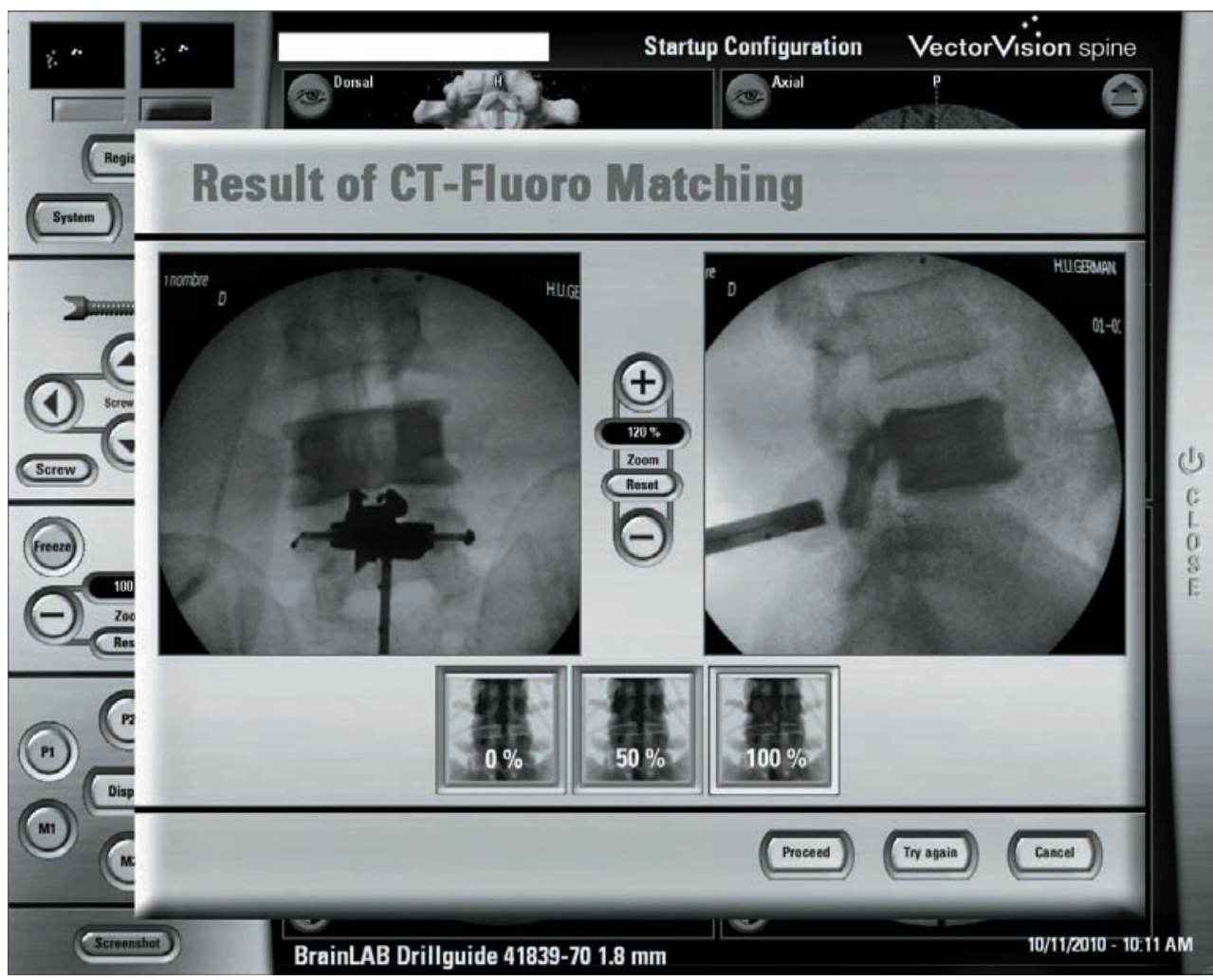

Figura 2. Fusión imagenes TC y 2D intraoperatorias.

mless $^{29}$ a nivel cervical, cirugía guiada con Fluoroscopio y $\mathrm{TC}^{9,36}, \mathrm{RM}^{8}$, monitorización neurofisiológica ${ }^{11}$, endoscopia asistida $^{2}$ y la detección con ultrasonido ${ }^{22}$ del trayecto de tornillo pedicular. Todos ellos permiten mejorar la precisión en la colocación del implante pero ninguno ofrece una imagen real-virtual directa del trayecto de los tornillos hasta la introducción de la navegación.

La navegación, fue aplicada en la columna ${ }^{9,24}$ a partir del año 2000, pero debido a una limitación de los software y hardware, a la lentitud del procedimiento, por una curva de aprendizaje difícil y por la relación beneficio coste económico, no fue integrada en el arsenal tecnológico para la cirugía de columna. Desde el 2007 y después de mejorar dichos aspectos, la navegación ha experimentado una expansión de su uso. Se han descrito diferentes sistemas de navegación para la fijación de columna según el método y obtención de imágenes radiológicas sea con Fluoro 2DTC, Fluoro ISO C-3D, CT y RMN, esta última la menos consolidada, siendo las diferencias de precisión entre ellos insignificantes $^{34,26,15}$. (Tabla 1).

El objetivo de este trabajo es presentar los resultados obtenidos, principalmente en cuanto a la precisión del implante en una serie prospectiva de 121 pacientes intervenidos por un mismo equipo entre el Servicio de Neurocirugía del H.G.T.P. de Badalona y Centro Médico Teknon.

Tabla 2

Valoración del implante según la escala tomográfica de Heary

\begin{tabular}{|l|l|}
\hline Grado & \multicolumn{1}{|c|}{ Descripción } \\
\hline I & Tornillo completamente contenido en el pedículo \\
\hline II & Tornillo viola la pared lateral del pedículo pero la punta está totalmente contenida en el cuerpo vertebral \\
\hline III & Tornillo perfora la pared lateral del pedículo \\
\hline IV & Perforación de la pared medial o inferior del pedículo \\
\hline V & Viola el cuerpo vertebral y pone en peligro la médula espinal, raíz o vasos; requiriendo reintervención \\
\hline
\end{tabular}




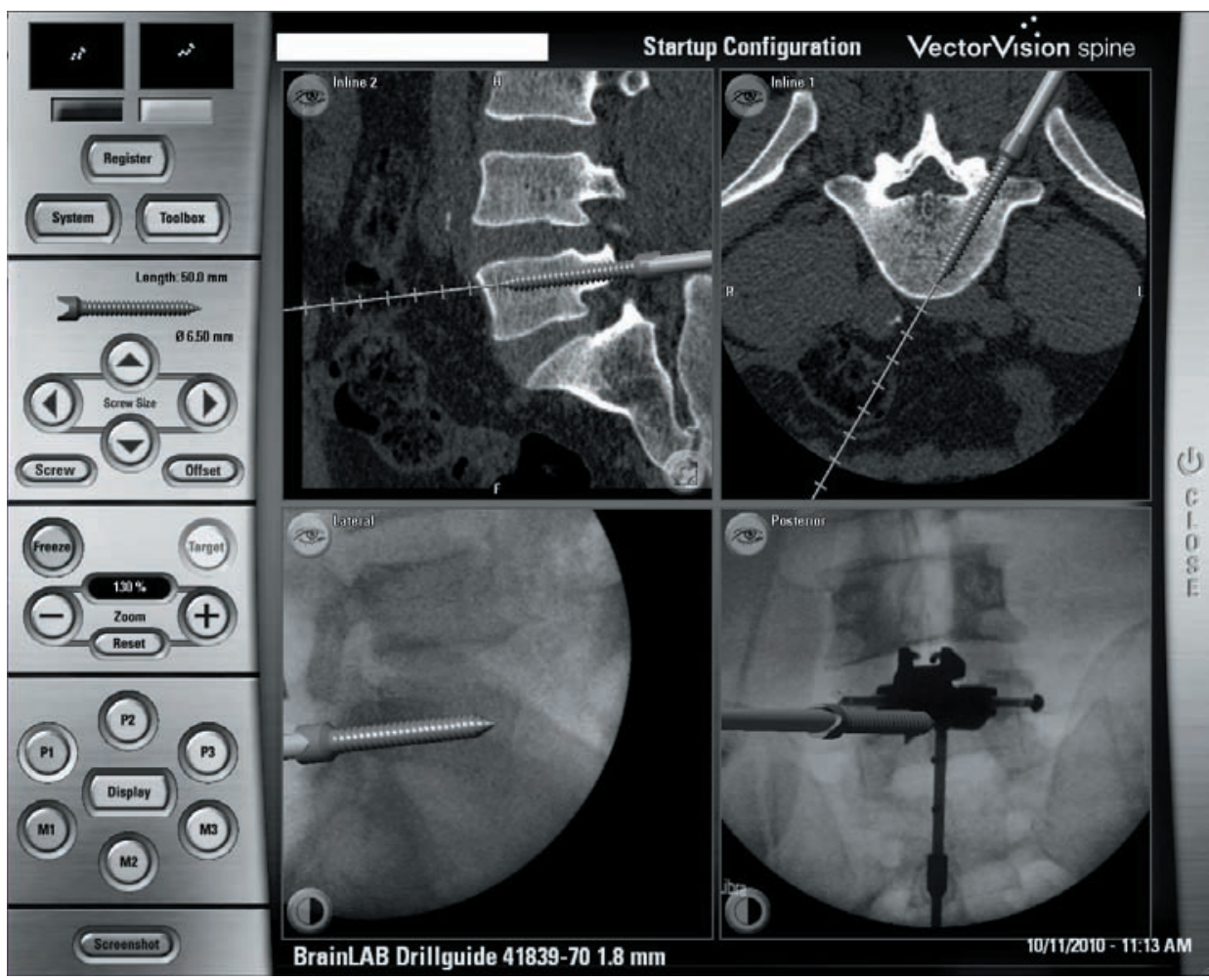

Figura 3. Navegación en tiempo real-virtual.

\section{Material y método}

Se trata de una muestra de 121 casos con indicación de una instrumentación vertebral posterior por diferentes patologías y niveles lesionales, utilizando para la navegación el modelo Kolibrí y Vector Visión (Brain LAB, Feldkirchen, Germany).

A todos los pacientes se les realizó un TC preoperatorio según protocolo de navegación, consistente en cortes axiales de $1,5 \mathrm{~mm}$ tanto del nivel lesional como los inmediatos adyacentes con el objetivo de obtener imágenes limitadas al marco anatómico de los espacios vertebrales a tratar. El paciente es colocado sobre la mesa de intervenciones en posición prono y una vez preparado el campo quirúrgico se marca con Rx el nivel lesional para colocar una pinza en la apófisis espinosa como soporte de las esferas reflectantes que serán captadas por la cámara foto-óptica del navegador. A continuación con el fluoroscopio 2D, al cual se le adapta un Kit. de fluoroscopia con múltiples puntos referenciales, se adquiere una imagen en AP (Fig. 1) que es transferida directamente al navegador, donde se comprueba la precisión anatómica con un instrumento precalibrado y dicha precisión no debe superar los $1,5 \mathrm{~mm}$ de desviación. Se repite el mismo procedimiento en una proyección lateral, a continuación con las imágenes obtenidas se realiza una fusión (Fig. 2) con las de la TC preoperatoria previamente introducidas al navegador obteniendo una imagen ósea tridimensional de alta calidad que nos permite navegar en tiempo real virtual (Fig. 3).

A nivel cervical y dorsal, debido a las dificultades técnicas para la obtención de buenas imágenes radiológicas sólo se ha navegado con las imágenes de la TC preoperatoria necesitando tomar diferentes marcas anatómicas intraoperatorias en la vértebra o vértebras a intervenir según protocolo incluido en el software del navegador (Fig. 4.1, 4.2, 4.3).

Para valorar los resultados de la precisión del implante se ha comparado las imágenes de planificación de todos los pacientes, almacenadas en el disco duro del navegador, con los resultados de la TC postoperatoria realizada también según protocolo de navegación. Con la escala de Heary ${ }^{17}$ (Tabla 2) se ha clasificado la situación del tornillo y además también se ha valorado el tiempo del registro y calibraje del instrumental, el tiempo total del procedimiento, la exposición radiológica según número de vistas o disparos con el Fluoro 2D y la valoración clínica mediante las escalas de VAS, índice de discapacidad de Oswesrty y JOA(L) así como el grado de satisfacción con el procedimiento.

\section{Resultados}

La serie de 121 casos se distribuyó en 68 varones y 53 mujeres con una edad media de 50,35 años (Tabla 3). Se implantaron 580 tornillos (Tabla 4) distribuidos en 480 


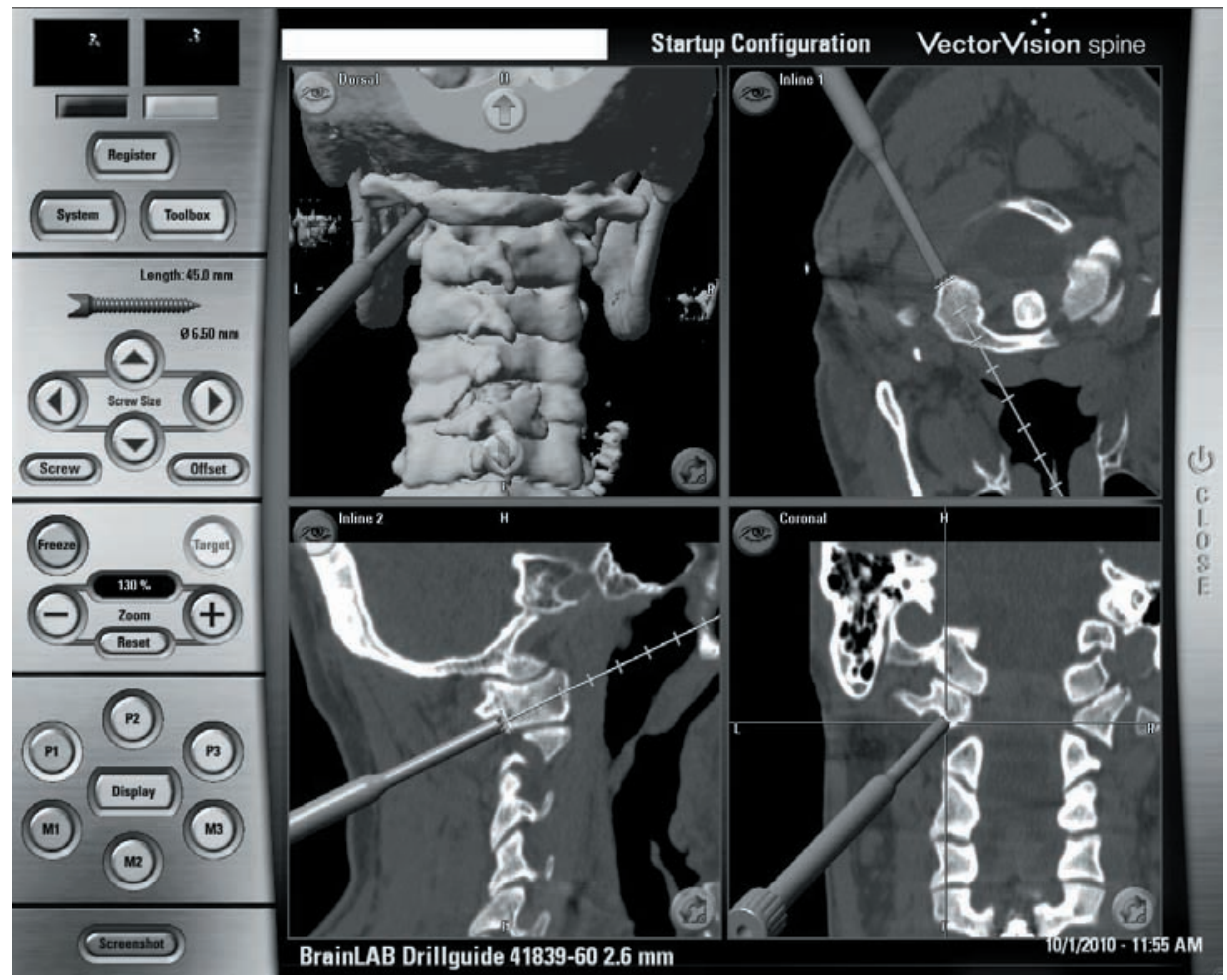

Figura 4.1. Navegación articular en Cl con TC.

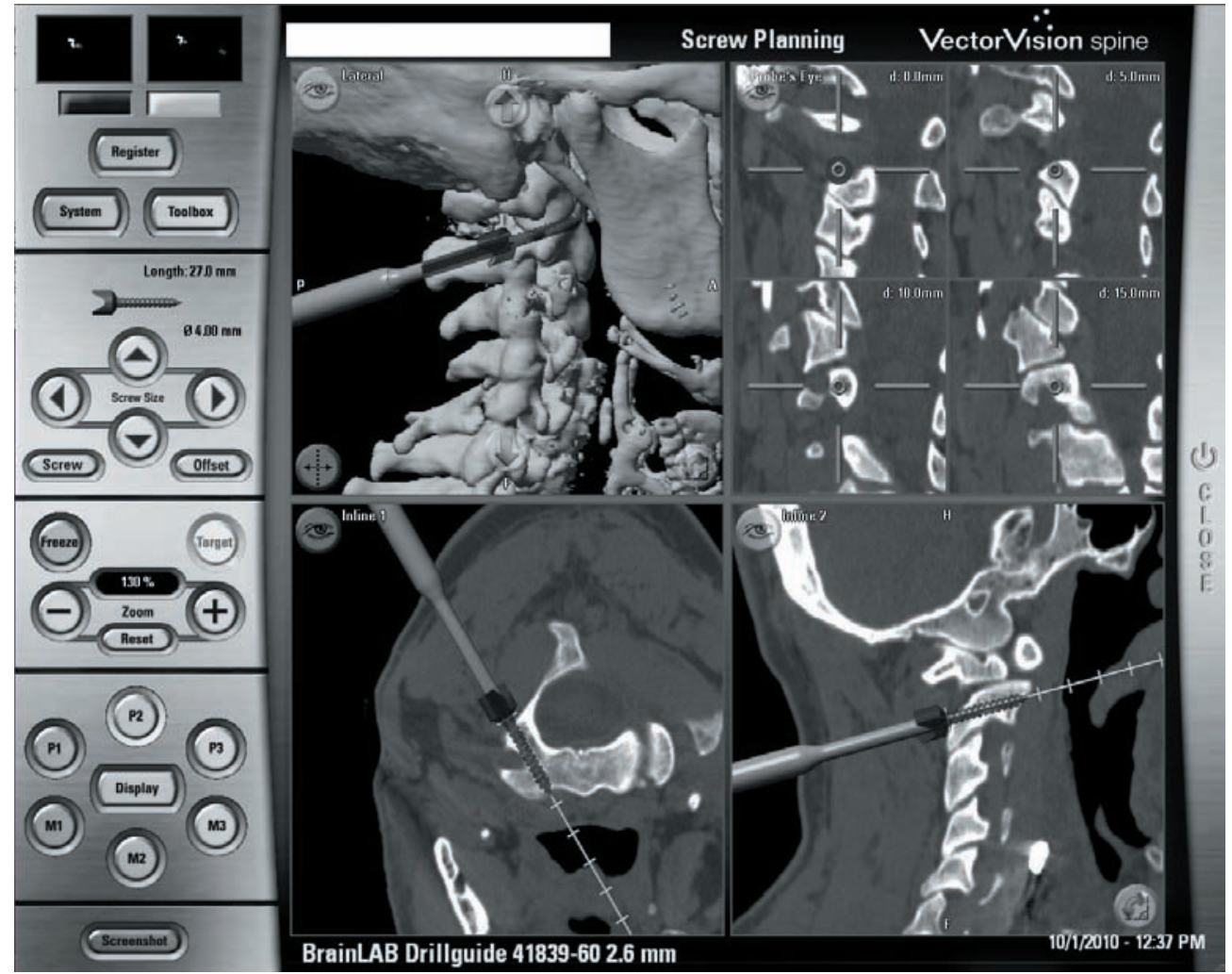

Figura 4.2. Navegación pedicular en C2 con TC. 


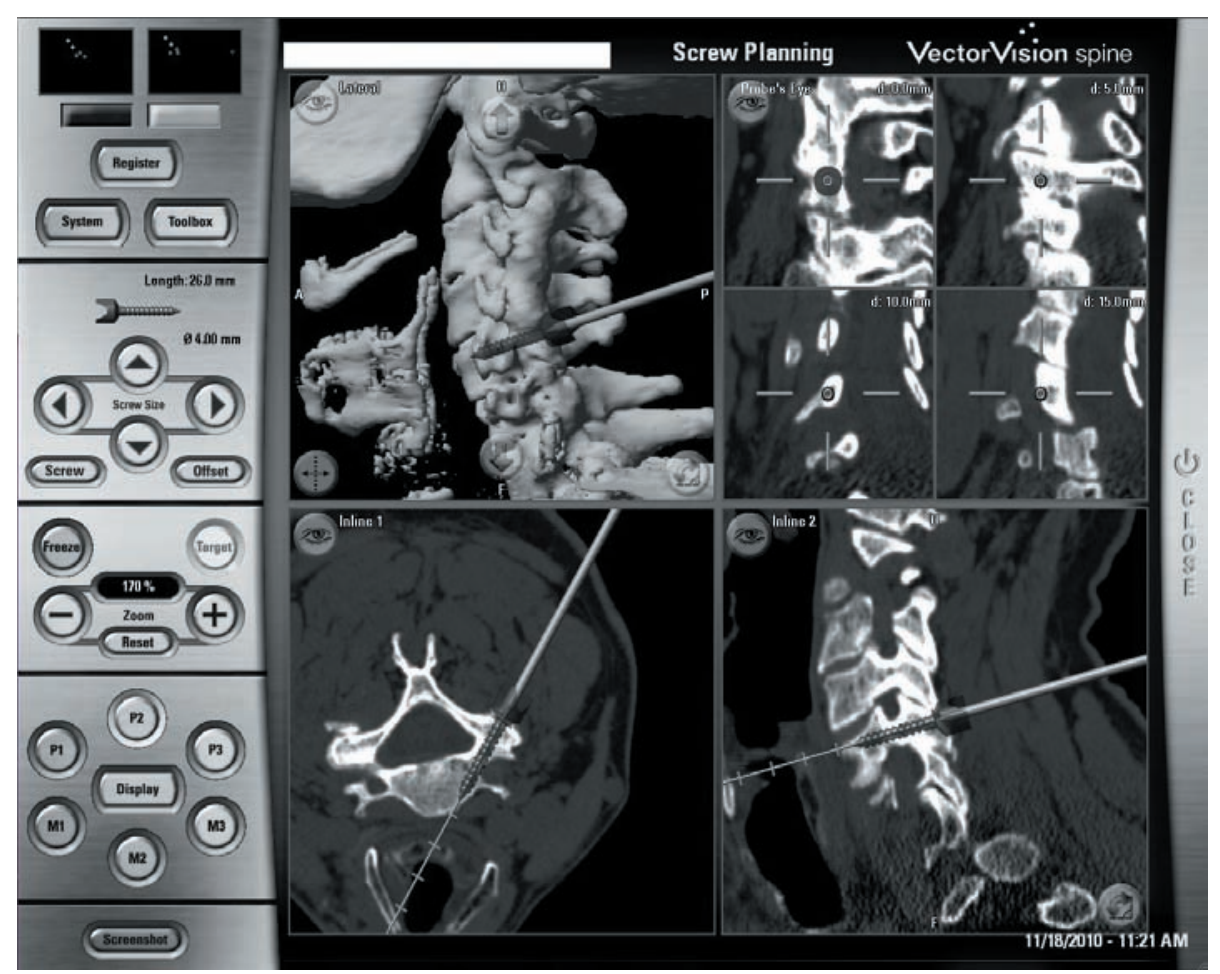

Figura 4.3. Navegación pedicular en C5 con TC.

lumbo-sacros, 38 dorsales y 62 cervicales, de estos últimos 24 en el segmento C1-C2. Todos los tornillos fueron pediculares a excepción de los 12 articulares de C1. La téc-

\section{Tabla 3}

Datos generales

Datos Valores

Total pacientes

121

Edad (media)

50.35 años

Sexo $(\mathrm{M} / \mathrm{F})$

$68 \mathrm{M} / 53 \mathrm{~F}$

Niveles intervenidos

Cervical

Dorsal

Lumbosacro

Patología degenerativa

Patología inflamatoria

Patología tumoral

Patología traumática

Estadía hospitalaria (media) nica quirúrgica empleada (Tabla 5) fue abordaje percutáneo en 51 pacientes, MIS en 28 y 42 a cielo abierto empleando una fijación rígida en 44 casos y semi-rígida o dinámica en 77 según patología del paciente.

La estancia media de los pacientes fue de 5,8 días, el tiempo medio quirúrgico de $3 \mathrm{~h} 14 \mathrm{~m}$. y la exposición radiológica fue de 1 a 2 disparos para localizar el nivel en región cervical y dorsal y 2 disparos en región lumbar. El tiempo medio de registro del instrumental fue de 2'49".

En la valoración por TC postquirúrgica, según escala de grados de Heary, 571 tornillos fueron intrapediculares sin violación de pared, 6 con desviación lateral externa

Tabla 4

Distribución de tornillos por región

\begin{tabular}{rlc}
170 & Región & $\mathbf{N}^{\mathbf{0}}$ Tornillos \\
22 & Cervical & 62 \\
12 & Dorsal & 38 \\
108 & Lumbar & 370 \\
1 & Sacra & 110 \\
4 & & \\
8 & Total & $\mathbf{5 8 0}$ \\
\hline 87 días & &
\end{tabular}




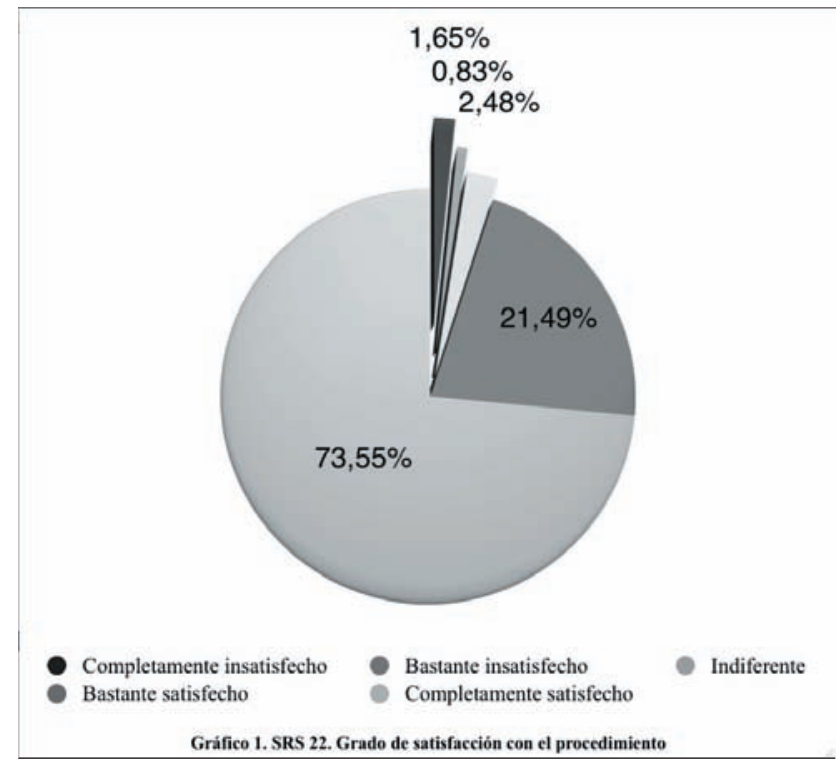

y 3 con desviación medial considerándose finalmente un $98,45 \%$ de fiabilidad de la planificación con navegación. Como complicaciones (Tabla 6) una reintervención por dolor radicular debido a una mala posición del tornillo a nivel cervical, 1 fractura de un tornillo lumbar y 3 casos de infección quirúrgica.

La valoración (Tabla 7) del VAS, Oswestry y JOA (L) del preoperatorio respecto al mes de la cirugía fue de $8.6 / 3.0,68.0 \% / 23.0 \%$ y $6,4 \% / 13,1 \%$ respectivamente

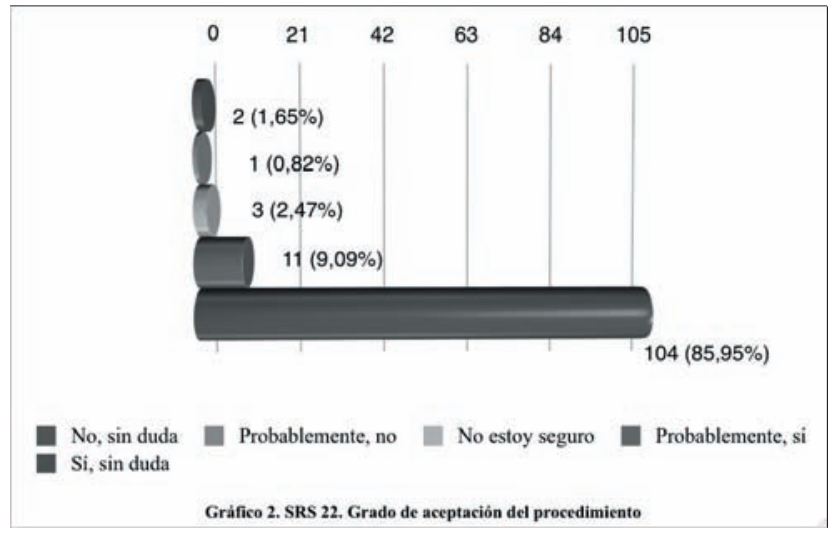

manteniéndose está mejoría con poca desviación a los 3,6 y 12 meses en 53 pacientes controlados al año. En cuanto al grado de satisfacción con el procedimiento fue de un 94.9\% (Gráfico 1) entre completamente y muy satisfecho

Tabla 5

Procedimiento quirúrgico

\begin{tabular}{lccc}
\hline $\begin{array}{l}\text { Tipo de } \\
\text { abordaje }\end{array}$ & $\begin{array}{c}\mathbf{N}^{\mathbf{0}} \\
\text { pacientes }\end{array}$ & $\begin{array}{c}\text { Inst. } \\
\text { rígida }\end{array}$ & $\begin{array}{c}\text { Inst. } \\
\text { semirrígida }\end{array}$ \\
\hline Abierto & 42 & 33 & 9 \\
MIS & 28 & 8 & 20 \\
Percutáneo & 51 & 3 & 48 \\
Total & $\mathbf{1 2 1}$ & $\mathbf{4 4}$ & $\mathbf{7 7}$ \\
\hline
\end{tabular}

Tabla 6

Complicaciones

\begin{tabular}{|c|c|c|c|c|c|}
\hline \multicolumn{4}{|l|}{ Evento } & \multirow[t]{2}{*}{$\mathbf{N}^{\mathbf{o}}$} & \\
\hline \multicolumn{4}{|c|}{ Desviación de tornillos: (sin necesidad de reintervención) } & & $9(1.55 \%)$ \\
\hline \multicolumn{6}{|c|}{$\begin{array}{l}\text { Grado II: } 2 \text { (1 cervical, } 1 \text { lumbar) } \\
\text { Grado III: } 4 \text { (cervical 1, dorsal 2, lumbar 1) } \\
\text { Grado IV: } 3 \text { (cervical 1, lumbar, } 2 \\
\text { (Escala tomográfica de Heary) }\end{array}$} \\
\hline \multicolumn{5}{|c|}{ Reintervención } & $1(0.8 \%)$ \\
\hline \multicolumn{5}{|c|}{ Fractura de tornillo } & $1(0.17 \%)$ \\
\hline \multicolumn{5}{|c|}{ Infección postquirúrgica } & $3(2.4 \%)$ \\
\hline \multicolumn{5}{|c|}{ Cefalea postural (Hipotens LCR-TC OK) IQ percutánea } & $1(0.8 \%)$ \\
\hline \multicolumn{6}{|c|}{$\begin{array}{c}\text { Tabla } 7 \\
\text { Valoración clínica }\end{array}$} \\
\hline Escalas & $\begin{array}{l}\text { Preoperatorio } \\
\quad(\mathrm{N}=121)\end{array}$ & $\begin{array}{l}\text { Post } 1 \text { mes } \\
(\mathrm{N}=121)\end{array}$ & $\begin{array}{l}\text { Post } 3 \text { mes } \\
(\mathrm{N}=100)\end{array}$ & $\begin{array}{l}\text { Post } 6 \text { mes } \\
\quad(N=87)\end{array}$ & $\begin{array}{l}\text { Post } 12 \text { mes } \\
(\mathrm{N}=53)\end{array}$ \\
\hline VAS & $8.6 / 1.8$ & $3.0 / 0.7$ & $3.0 / 0.7$ & $3.05 / 0.72$ & $2.9 / 0.6$ \\
\hline Oswestry & $68.0 \%$ & $23.0 \%$ & $23.0 \%$ & $23.0 \%$ & $22.0 \%$ \\
\hline $\mathrm{JOA}(\mathrm{L})$ & 6.46 & 3.11 & 13.11 & 3.09 & 13.19 \\
\hline
\end{tabular}


que coincidió con el grado de aceptación en el supuesto de repetir el tratamiento. (Gráfico 2).

\section{Discusión}

La fijación vertebral posterior es un técnica muy experimentada con alto índice de precisión y grado de evidencia motivo por el cual, condiciona a cirujanos de columna a prescindir de la navegación. A pesar de que la navegación fue introducida a principios del año 2000, su uso no tomó auge hasta el 2007 coincidiendo con las modificaciones técnicas introducidas en el software y hardware del navegador, que han facilitado y mejorado el global del procedimiento de navegación. El índice de precisión del implante con navegación, en nuestra serie, ha sido del 98,4\% similar a la de otros autores siendo, entre otras ventajas, superior a las técnicas convencionales ${ }^{17,11,16}$. La valoración del implante se ha realizado mediante el control de una TC postoperatoria en más de 100 casos (Fig. 5) y aplicando la escala de Heary cuyos resultados se compararon con la planificación del trayecto de todos los casos almacenados en la memoria del navegador.

Como se ha mencionado, existen diferentes opciones de navegación y entre ellas la navegación con Fluoro 2D-TC utilizada para este trabajo, la Fluoro ISO-C 3D y la navegación por TC. Todas ellas tienen en común la necesidad de asociarse a un navegador y que reducen la desviación del implante por debajo del $10 \%$ así como el número de reintervenciones ${ }^{17}$. Se diferencian principalmente por la adquisición de las imágenes ya que con el 3D como con la $\mathrm{TC}$, estas son adquiridas directamente en el quirófano y en la misma posición quirúrgica del paciente. Con la Fluoro 2D-TC la adquisición se realiza previamente a la cirugía y en posición supina. Se ha mencionado que la diferente posición entre la adquisición de imagen y la quirúrgica podría influir en la planificación de la navegación 25 lo cual no ha sucedido con nuestro sistema ya que el software permite adaptar ambas posiciones sin presentar ninguna dificultad en la planificación y navegación. Entre otras diferencias técnicas, tanto con la Fluoro ISO C-3D como con la TC al adquirir las imágenes de forma directa permiten la posibilidad de corregir los errores del implante en el mismo acto quirúrgico si fuera necesario. A favor de la Fluoro 2D respecto a los otros sistemas ${ }^{25}$ está la posibilidad de adquirir mayor campo volumétrico con menor irradiación y coste económico además de no precisar de una mesa quirúrgica complementaria absolutamente radiotransparente.

La dificultad de la adquisición de buenas imágenes con el Fluoro 2D tanto a nivel cervical como dorsal dificulta una correcta fusión con las imágenes de TC lo que obliga a navegar solo con TC mediante la adquisición de múltiples puntos de referencia bajo cirugía abierta y según protocolo determinado por el sistema de navegación. Esta dificultad

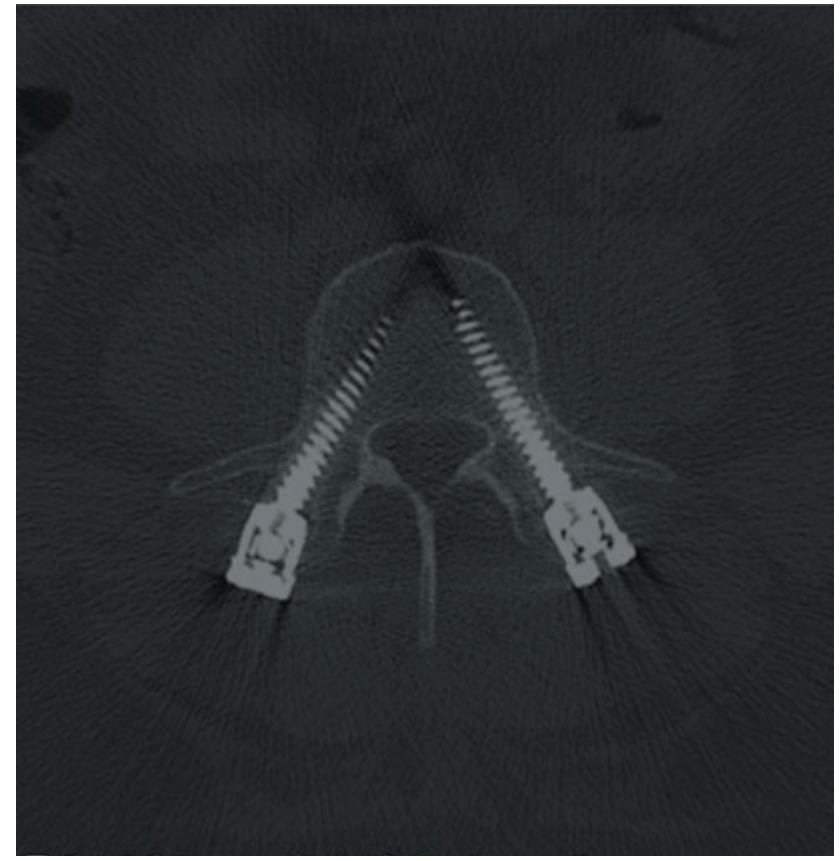

Figura 5. Control TC postquirúrgico con grado I de Heary del implante.

en la adquisición de imagen, el diseño del instrumental y la naturaleza de la patología condicionó el tipo de cirugía realizada en nuestra serie, 42 casos a cielo abierto, 28 casos con abordajes mínimamente invasivos y 51 casos con técnica percutánea poco referida en la bibliografía con navegación.

A nivel cervical la cirugía se realizó a cielo abierto con la detección de múltiples marcas anatómicas según TC . Las dificultades y el riesgo de una fijación cervical pedicular posterior ${ }^{16,19}$, principalmente en el segmento C1$\mathrm{C} 2$, por posibles lesiones neurológicas o vasculares la convierten en una técnica de riesgo incluso en manos expertas pero con la aplicación del navegador estas complicaciones han disminuido, permitiendo una mayor seguridad del procedimiento y una menor curva de aprendizaje para los cirujanos no expertos. De los 6 casos intervenidos en C1-C2 ninguno presentó complicaciones y los tornillos se alojaron según la planificación a excepción de una desviación lateral interna y otra lateral externa sin repercusión clínica. En este segmento por sus características anatómicas y al predominar patologías con inestabilidad se aconseja poner el clamp de referencia para navegación en C2 lo que hicimos en dos casos de la serie mientras en el resto se individualizó cada vértebra no encontrando diferencias apreciables de precisión en la navegación. Con otros sistemas de navegación se coloca el clamp en el arco de fijación de Mayfield para navegar. Hay autores que han descrito cirugía guiada directa para fijación transarticular C1-C2 con el Fluoro ISO $\mathrm{C}-3 \mathrm{D}^{33}$. 
En la columna cervical media y baja clásicamente se introducen tornillos articulares pero la navegación permite implantar tornillos pediculares lo que confiere una mayor estabilidad biomecánica con menor riesgo de complicaciones $^{19}$.

De los 68 tornillos de la serie solo uno presentó una desviación lateral interna que se manifestó con dolor radicular siendo necesario una reintervención, la única de la serie, para extracción del mismo. Se ha descrito que la precisión del implante a nivel cervical con navegador ${ }^{27}$ ( Fluoro 2D-TC) frente a la sin navegador en manos expertas es del $97 \%$ y $91,7 \%$ respectivamente además de reducir el tiempo quirúrgico.

A nivel cérvico dorsal las dificultades de una fijación son bien conocidas por la escasa visualización radiológica debido a la interposición de los hombros y la disposición anatómica del segmento dorsal alto. La cirugía guiada con navegador elimina estos obstáculos al poder navegar solo con las imágenes de la TC preoperatoria permitiendo una correcta precisión del implante como sucedió en los pacientes de la serie.

A nivel dorsal, las indicaciones quirúrgicas de fijación más frecuente son las fracturas, aplastamientos y lesiones tumorales y para conseguir una fijación biomecánicamente estable y rígida debe extenderse a dos niveles adyacentes de la vértebra o vértebras lesionadas las cuales deben estar incluidas en el estudio TC prequirúrgico. Esta cirugía se realiza de forma abierta y se guía por TC utilizando la Fluoro 2D tan solo para marcar el nivel quirúrgico y en cambio con el sistema 3D y TC intra-operatorio se precisa más de una toma de imágenes por la limitación volumétrica mencionada y por lo tanto más irradiación.

La variabilidad de las dimensiones de los pedículos de las vértebras dorsales ${ }^{17,26}$ y las alteraciones en la posición de las vértebras en determinadas patologías como por ejemplo en la escoliosis son causa de mal posicionamiento de tornillos ${ }^{20,28,35,31,14}(3-42 \%)$ con serias complicaciones tanto neurológicas como pleurales y vasculares principalmente en el segmento D4-D9, las cuales podrían evitarse con el uso de la navegación. En nuestra serie de 38 tornillos no hubo ninguna complicación y todos fueron implantados según se planificaron y con un tiempo quirúrgico reducido. Al igual que en el segmento cervical, por su menor incidencia, la navegación aporta una confianza y seguridad a cirujanos poco expertos, difícil de conseguir con el método clásico como se demuestra en estudios comparativos ${ }^{10}$ entre ambas técnicas, aportando $85 \%$ de tornillos bien colocados sin navegación precisando reintervención un 7\% frente al 95\% con navegación y sin ninguna reintervención.

A nivel lumbar es donde existe mayor experiencia del uso del navegador por ser mayor la frecuencia de patología principalmente de origen degenerativo. La cirugía se realiza mediante la fusión de imágenes intra-operatorias adqui-

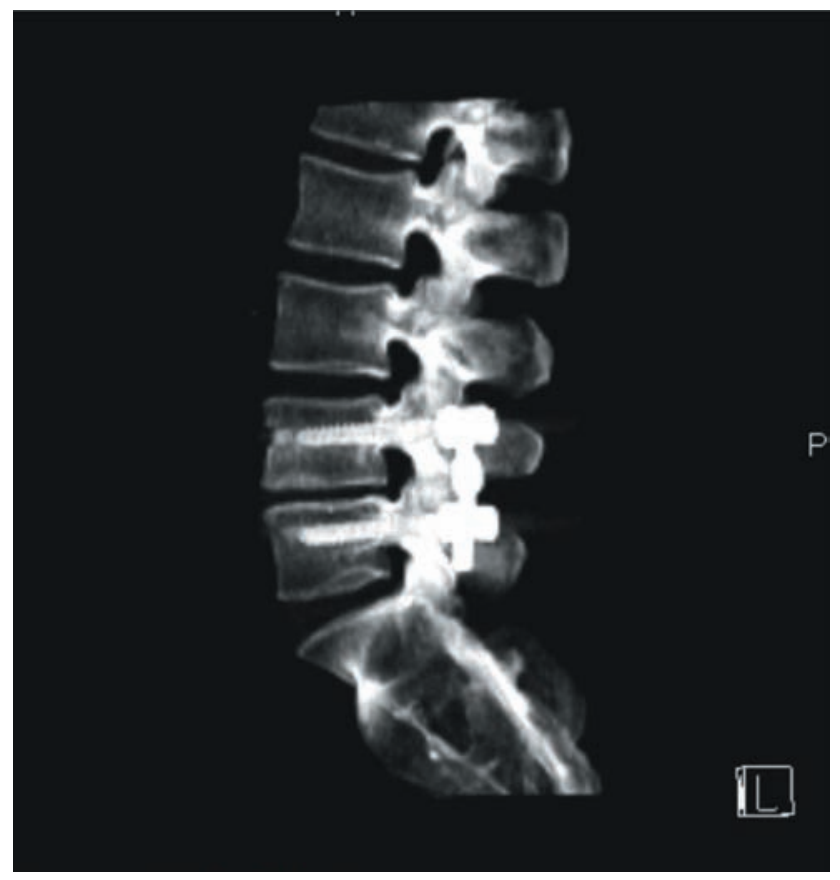

Figura 6. Control sagital fijación percutanea dinámica.

ridas con el Fluoro 2D e imágenes del TC prequirúrgico, permitiendo realizar cirugía guiada con técnica abierta (42 casos) mini-open (28 casos) o percutánea (51 casos).

El instrumental que utilizamos para la localización y perforación del pedículo está precalibrado y en cuanto al sistema de fijación a emplear no hay limitación ya que podemos calibrarlo con la matriz de calibración y así poder usar diferentes sistemas de fijación siempre con tornillos canulados.

La técnica percutánea la hemos realizado en pacientes con indicación de fijación a un solo nivel bien sea por una inestabilidad ligamentaria grado I y/o discopatía con o sin Módic, utilizando para ello instrumental dinámico (Fig. 6) o semi-rígido. Cuando la indicación sobrepasa más de un segmento hemos realizado técnica mini-open (28 casos) con incisiones paralelas para-vertebrales según técnica de Wiltse. En estas técnicas MIN el clamp se coloca en la espinosa seleccionada con una mínima incisión en línea media.

Los resultados obtenidos en relación a la precisión del implante han sido de $98,45 \%$ en 571 tornillos según escala de Heary con una desviación de 9 tornillos (1,55\%). La desviación de un tornillo a nivel lumbar, ${ }^{1,18}$ es variable y en relación a la experiencia del cirujano siendo la desviación del mismo la más referida con cifras ${ }^{17}$ desde un $6,5 \%$ o de un $11,4 \%$ al $42,3 \%$ sin navegación frente a $4,3 \%$ al $6,8 \%$ con Fluoro-CT o del 0,0 al 3,6\% con Fluoro ISOC 3D . La mayoría de los tornillos desviados (85\%-90\%) son clínicamente asintomáticos pero pueden influir en la biomecánica de la fijación o fusión a largo plazo. 
Hemos intentado justificar la desviación de los tornillos de nuestra serie y para ello se revisó todo el procedimiento quirúrgico almacenado en el ordenador del navegador concluyendo que existen diferentes motivos para justificar dichas complicaciones y entre ellas mencionar nuestra curva de aprendizaje en los primeros 17 casos, la rotación grave y luxación de la vértebra a nivel $\mathrm{C} 1$ y por no navegar con el porta-tornillos calibrado en el intento de reducir el tiempo quirúrgico al confiar con la guía de la aguja Kirchner introducida en el pedículo. La corrección de algunos de estos factores nos ha ayudado a mejorar el índice de precisión de forma progresiva.

La duración del procedimiento quirúrgico con navegación ha sido, entre otros, uno de los motivos de aversión a la técnica por parte de cirujanos de columna de forma errónea ya que en estudios comparativos ${ }^{32}$ de cirugía con y sin navegación el tiempo es menor con navegación. En nuestra serie el tiempo que se ha utilizado para el registro y calibración del instrumental ha sido de 2'49" superada la curva de aprendizaje, lo cual no ha repercutido en el tiempo global del procedimiento sino más bien que lo reduce ya que facilita la búsqueda de los puntos de inserción y dirección del tornillo.

Entre las ventajas de la cirugía guiada con navegación, además de su precisión, es la escasa irradiación de todo el equipo quirúrgico y paciente. Los autores no han cuantificado la irradiación con dosímetro pero sí en número de disparos en proyección de perfil en región cervical y dorsal con una media de 1-2 respectivamente para localizar el nivel. A nivel lumbar, calibrado previamente el Kit. de fluoroscopia, se realizaron una media de 2 disparos por paciente cuando en un procedimiento sin navegación y principalmente percutáneo se necesita un mínimo de $4 \mathrm{o}$ más disparos por tornillo para comprobar el trayecto del mismo. La exposición a la irradiación ha sido reportada en estudio comparativo con y sin navegación ${ }^{21}$, siendo prácticamente inapreciable la irradiación con navegación versus sin navegación (12,4 mili-REM) del equipo quirúrgico así como del paciente.

También hemos podido comprobar, aunque no era el principal objetivo de este trabajo, la mejoría clínica postoperatoria del paciente tanto del dolor (VAS 3.0), como del índice de discapacidad de Owestry (23.0\%) JOA (L) 13,1 y grado de satisfacción con la técnica $(94,9 \%)$, lo cual se justifica por la menor agresividad tanto de las partes blandas como óseas del procedimiento.

La indicación de la navegación espinal va ampliándose a otros campos, además de la instrumentación, como la realización de vertebroplastia ${ }^{12,30}$, guía en la artroplastia lumbar ${ }^{37}$ , resección de osteofitos en cirugía por vía anterior ${ }^{37}$ y posibilidad de fijación anterior de odontoides ${ }^{5}$. Los autores la han utilizado en biopsias guiadas tumoral cervical y como guía en cirugía MIS para el implante intersomático.

\section{Conclusiones}

Superada la curva de aprendizaje de la técnica, la navegación aporta al procedimiento de la instrumentación vertebral posterior una mayor seguridad, una disminución del riesgo de lesión neurológica, vascular y de irradiación y facilita el aprendizaje para jóvenes cirujanos convirtiendo la técnica en un acto elegante, mínimamente invasivo e incluso de menor duración que un procedimiento convencional.

\section{Bibliografia}

1. Amiot, L.P., Lang, K., Putzier M.: Comparative Results Between Conventional and Computer-Assisted Pedicle Screw Installation in the Thoracic, Lumbar, and Sacral Spine. Spine 2000; 25: 606-614.

2. Assaker, R., Cinquin, P., Cotton., Lejeune, J.P.: Imageguided endoscopic spine surgery:Part I. A feasibility study. Spine 2001; 26: 1705-1710.

3. Baldauf, J., Müller, J.U., Fleco, S., Hinz, P., Chiriac, A., Schroeder, H.W.: The value of intraoperative three dimensional fluoroscopy in anterior decompressive surgery of the cervical spine. Zentralbl Neurochir. 2008; 69: 30-34.

4. Balzer, J.R., Rose, R.D., Welch, W.C., Sclabassi, R.J.: Simultaneous somatosensory evoked potential and electromyographic recordings during lumbosacral decompresssion and instrumentation. Neurosurgery 1988; 42: 1318-1324; discussion 1324-1325.

5. Battaglia, T.C., Tannoury, T., Crowl, A.C., Chan, D.P., Anderson, D.G.: A cadaveric study comparing standard fluoroscopy with fluoroscopy-based computer navigation for screw fixation of the odontoid. J Surg Orthop Adv. 2005; 14: 175-178.

6. Bauer., Kerschbaumer., Poisel.: Cirugía Ortopédica, Columna. Edición original Georg Thieme Verlag; Marban Libros, Edición Española 1998.

7. Florensa, R., Noboa, R., Muñoz, J., et al.: Complicaciones de la artrodesis transpedicular lumbar. Neurocirugía 2001; 12: 216.

8. Florensa, R., Noboa, R., Muñoz, J., et al.: Results of C1-C2 transarticular screw fixation in a series of 20 patients. Neurocirugia 2002; 13: 429-435; discussion 436.

9. Foley, K.T., Simon, D.A., Rampersaud, Y.R.: Virtual fluoroscopy:computer-assited fluoroscopy navigation. Spine 2001; 15; 26: 347-351.

10. Fritsch, E., Duchow, J., Seil, R., Grunwald, I., Reith, W.: Accuracy of fluoroscopic navigation of pedicle screws. CT-based evaluation of bone screw placement. Orthopade. 2002; 31: 385-391. German.

11. Fuster, S., Vega, A., Barrios, G., Urdaneta, I., et al.: Fiabilidad del navegador en la colocación de tornillos pediculares toracolumbares. Neurocirugía 2010; 21: 306-311. 
12. Gordon., Deen, H.G., Nottmeier, E.W.: Balloon kyphoplasty for treatment of sacral insufficiency fractures. Report of three cases. Neurosurg Focus. 2005; 18: E7.

13. Heary, R.F., Bono, C.M., Black, M.: Thoracic pedicle screwspostoperative computerized tomography scanning assesment. J. Neurosurg 2004; 100 (suppl Spine): 325-331.

14. Heini, P., Scholl, E., Wyler, D., et al.: Fatal cardiac tamponade associated with posterior spinal instrumentation: a case report. Spine 1998; 23: 2226-2230.

15. Holly, L.T., Foley, K.T.: Intraoperative spinal navigation. Spine (Phila Pa 1976). 2003 ;28 (15 Suppl): S54-61.

16. Hott, J.S., Papadopoulos, S.M., Theodore, N., Dickman, C.A., Sonntag, V.K.: Intraoperative Iso-C C-arm navigation in cervical spinal surgery: review of the first 52 cases. Spine (Phila Pa 1976) 2004; 29: 2856-2860.

17. Jaehon, M., Kim,,M.D., Andrea, L., Bowers,,M.D., and Kingsley, R., Chin, M.D.: Intraoperative Imaging Techniques in Spine Surgery. J. Spinal Disord. Tech. 2007: 20: 78-86.

18. Jutte, P.C., Castelein, R.M., et al.: Complications of pedicle screws in lumbar and lumbosacral fusions in 105 consecutive primary operations. Eur Spine J 2002; 11: 594-598.

19. Kast, E., Mohr, K., Ricther, H.P., et al.: Complications of transpedicular screw fixation in the cervical spine. Eur Spine J 2006; 15: 327-334.

20. Kim, Y., Lenke, L., Bridwell, K.: Free Hand Pedicle Screw Placement in the Thoracic Spine: It is Safe? Spine 2004; 29: 333-342.

21. Kim, C.W., Lee, Y.P., Taylor, W., Oygar, A., Kim, W.K.: Use of navigation-assisted fluoroscopy to decrease radiation exposure during minimally invasive spine surgery. Spine J. 2008; 8: 584-590. Epub 2007 Feb 20.

22. Koller, H., Hitzl, W, Acosta, F.: In vitro study of accuracy of cervical pedicle screw insertion using an electronic conductivity device (ATPS part III). Eur Spine J. 2009; 18 : 1300-1313. Epub 2009 Jul 3

23. Kosmopoulos, V., Schizas, C.: Pedicle screw placement accuracy: meta-analysis. Spine (Phila Pa 1976). 2007; 32: E111-120

24. Langston, T., Holly, M.D., and Kevin, T., Foley, M.D.: Intraoperative Spinal Navigation. Spine 2003; 28: 554-561.

25. Le Huec, J.C., Aunoble,S., Chalali, M., Bourghli, A.: Navigation and CT scan for navigation in spinal surgery. Oarm possibilities and limits. Argos spine News\&Journal. 2010; $22 \mathrm{~N}^{\mathrm{o}} 1$ Focus 42.

26. Lekovic, G.P., Potes, E.A., Karahalios, D.G., Hall, G.A.: Comparison of two techniques image-guided thoracic pedicle screw placement: a retrospective study of 37 patients and 277 pedicle screws. J Neurosurg Spine. 2007; 7: 393398.

27. Liu, Y.J., Tian, W., Liu, B., et al.: Accuracy of CTbased navigation of pedicle screws implantation in the cervical spine compared with X-ray fluoroscopy technique. Zhonghua Wai Ke Za Zhi. 2005; 43): 1328-1330.
28. Metz, L.N., Burch, S.: Computer-assisted surgical planning and image-guided surgical navigation in refractory adult scoliosis surgery: case report and review of the literature. Spine (Phila Pa 1976). 2008; 33: E 287-292.

29. Nolte, L., Zamorano, L., Arm, E., et al.: Image guided computer-assisited spine surgery:a pilot study on pedicle screw fixation. Stereotactic Funct Neurosurg 1996; 66: 108117

30. Ohnsorge, J.A., Siebert, C.H., Schkommodau, E., Mahnken, A.H., Prescher, A., Weisskopf, M.: Minimallyinvasive computer-assisted fluoroscopic navigation for kyphoplasty. Z Orthop Ihre Grenzgeb. 2005;143: 195-203. German.

31. Papin, P., Arler, V., Marchesi, D., et al.: Unusual presentation of spinal cord compression related to misplaced pedicle screws in thoracic scoliosis. Eur Spine J 1999; 8:156159.

32. Quiñones-Hinojosa, A., Robert Kolen, E., Jun, P., Rosenberg, W.S., Weinstein, P.R.: Accuracy over space and time of computer-assisted fluoroscopic navigation in the lumbar spine in vivo. J Spinal Disord Tech. 2006; 19:109113.

33. Rajasekaran, S., Vidyadhara, S., Shetty, A.P.: Iso-C3D fluoroscopy-based navigation in direct pedicle screw fixation of Hangman fracture: a case report. J Spinal Disord Tech. 2007; 20: 616-619.

34. Reichle, E., Sellenschloh, K., Morlock, M., Eggers, C.: Placement of pedicle screws using different navigation systems. A laboratory trial with 12 spinal preparations. Orthopade. 2002; 31: 368-371.

35. Sarlak, A.Y., Tosun, B., Atmaca, H., Sarisoy, H.T., Buluç, L.: Evaluation of thoracic pedicle screw placement in adolescent idiopatic scoliosis. Eur Spine J. 2009; 18: 18921897

36. Schlenzka, D., Laine, T., Lund, T.: Computer-assisted spine surgery: principles, technique, results and perspectives. Orthopade. 2000; 29: 658-669.

37. Smith, H.E., Vaccaro, A.R., Yuan, P.S., Papadopoulos, S., Sasso, R.: The use of computerized image guidance in lumbar disk arthroplasty. J Spinal Disord Tech. 2006; 19: 2227.

38. Woodard, E.J., Leon, S.P., Moriarty, T.M., Quinones, A., Zamani, A.A., Jolesz, F.A.: Initial experiencie with intraoperative magnetic resonace imaging in spine surgery. Spine 2001; 26: 410-417.

Florensa, R.; Muñoz, J.; Cardiel, I.; Bescós, A.; Tardáguila, M.; Plans, G.; Domínguez, C.J.; Colet, S.: Fijación vertebral posterior guiada por neuronavegación. Experiencia en 121 casos. Neurocirugía 2011; 22: 224-234.

Correspondencia: Dr. Ramon Florensa. Servicio de Neurocirugía. Hospital Germans Trias i Pujol . Badalona. E-mail: rflorensa.germanstrias@gencat.cat 